\title{
Comparative activity of carbapenem testing (the COMPACT study) in Turkey
}

\author{
Hakan Leblebicioglu ${ }^{1 *}$, Nedim Cakir ${ }^{2}$, Mustafa Celen ${ }^{3}$, Halil Kurt ${ }^{4}$, Hakan Baris ${ }^{5}$ and Joerg Laeuffer ${ }^{6}$, for \\ Turkish COMPACT Study Group
}

\begin{abstract}
Background: Recent evidence indicates that Gram-negative bacterial pathogens, the most common of which are Pseudomonas spp., Enterobacteriaceae, and Acinetobacter baumannii, are frequent causes of hospital-acquired infections. This study aims to evaluate the in vitro activity of doripenem and comparator carbapenem antibiotics against Gram-negative clinical isolates collected from COMParative Activity of Carbapenem Testing (COMPACT) study centres in Turkey.

Methods: Ten centres in Turkey were invited to submit Pseudomonas aeruginosa, Enterobacteriaceae, and other Gram-negative isolates from intensive care unit (ICU)/non-ICU patients with complicated intra-abdominal infections, bloodstream infections, or nosocomial pneumonia, including ventilator-associated pneumonia, between May and October 2008. Susceptibility was determined by each centre using E-test. A central laboratory performed species confirmation as well as limited susceptibility and quality-control testing.

Results: Five hundred and ninety six isolates were collected. MIC $_{90}$ values for doripenem, meropenem, and imipenem, respectively, were $32, \geq 64$, and $\geq 64 \mathrm{mg} / \mathrm{L}$ against Pseudomonas spp.; $0.12,0.12$, and $0.5 \mathrm{mg} / \mathrm{L}$ against Enterobacteriaceae; and $\geq 64 \mathrm{mg} / \mathrm{L}$ for each against other Gram-negative isolates. In determining the susceptibility of hospital isolates of selected Gram-negative pathogens to doripenem, imipenem, and meropenem, we found that against all pathogens combined, the $\mathrm{MIC}_{90}$ for ICU compared with non-ICU isolates was higher.
\end{abstract}

Conclusions: Doripenem showed similar or slightly better activity than meropenem and better activity than imipenem against the Gram-negative pathogens collected in Turkey.

\section{Background}

Modified treatment practices towards improving in-hospital patient care and reducing the development and spread of resistant strains begin with the surveillance of known infection-causing pathogens. Isolates of particular importance within the hospital setting are Pseudomonas spp. and Enterobacteriaceae, as well as other Gram negatives such as Acinetobacter baumannii (A.baumannii). Recent evidence indicates these pathogens are common causes of infection, including complicated intra-abdominal infection (cIAI), blood stream infection (BSI), and nosocomial pneumonia (NP). Escherichia coli (E. coli), for example, is the most common cause of BSIs in England, accounting for $18 \%$ of such infections [1].

\footnotetext{
* Correspondence: hakanomu@omu.edu.tr

'Ondokuzmayis University, Kurupelit Kampüsü, PK 55139, Kurupelit, Samsun, Turkey

Full list of author information is available at the end of the article
}

Surveillance results from the 2007 Meropenem Yearly Susceptibility Test Information Collection (MYSTIC) show ongoing resistance across Europe for these Gramnegative pathogens [2]. Acinetobacter spp. also play an increasing role in healthcare-associated infections [1]. In Turkey, for example, susceptibility of $A$. baumannii to imipenem decreased from $80.4 \%$ in 2000 to $40.0 \%$ in 2006 and from $71.7 \%$ to $40.0 \%$ for meropenem during the same time period [3].

Doripenem is a carbapenem antibiotic with activity similar to imipenem and ertapenem against Gram-positive cocci, and similar to meropenem against Gram-negative pathogens [4]. Doripenem was approved in the European Union on July 25,2008 , for NP, including ventilator-associated pneumonia (VAP), cIAIs, and complicated urinary tract infections [5].

The COMParative Activity of Carbapenem Testing (COMPACT) Study was conducted to evaluate the in
C Biomed Central

(c) 2012 Leblebicioglu et al; licensee BioMed Central Ltd. This is an Open Access article distributed under the terms of the Creative Commons Attribution License (http://creativecommons.org/licenses/by/2.0), which permits unrestricted use, distribution, and reproduction in any medium, provided the original work is properly cited. 
vitro activity of doripenem and comparator carbapenem antibiotics against recent Gram-negative clinical isolates; specifically Pseudomonas spp., Enterobacteriaceae, and other Gram negatives, including A. baumannii. This report focuses on the results from centres in Turkey and compares them with the general susceptibility pattern observed in COMPACT centres across Europe, the Middle East, and Africa.

\section{Methods}

Isolates of Pseudomonas aeruginosa, Enterobacteriaceae, and other Gram negatives were collected prospectively between May 2008 and June 2009 from 80 centres across 16 countries in Europe, the Middle East, and Africa, including 10 centres in Turkey. Each centre was asked to prospectively collect 60 non-duplicate Gramnegative isolates. Isolates were obtained from intensive care unit (ICU) and non-ICU patients hospitalised with 1 of 3 types of infection: cIAI, BSI, or NP, including VAP. Collecting centres determined susceptibility of the isolates to doripenem, imipenem, and meropenem using E-test strips according to the manufacturer's guidelines.

The study protocol was reviewed and approved by an independent ethics committee. The study was conducted in accordance with the principles in the Declaration of Helsinki and was consistent with applicable regulatory requirements.

Isolates were batched by each centre and sent to a reference laboratory (Quotient Bioresearch Ltd., Fordham, UK) for species confirmation. The reference laboratory determined the minimum inhibitory concentration (MIC) of doripenem, imipenem, and meropenem for all isolates identified by each centre's E-test as imipenem- or meropenem-resistant, according to the 2009 Clinical and Laboratory Standards Institute (CLSI) breakpoints, or as doripenem non-susceptible by the US Food and Drug Administration (FDA) breakpoints (Table 1) [6]. The MIC was determined by both broth microdilution using CLSI methodology [7] and E-test according to the manufacturer's methodology. Limited susceptibility testing was performed for quality control purposes on each centre's E-test results by randomly selecting $10 \%$ of the susceptible isolates from each centre. FDA breakpoints were used for doripenem since CLSI breakpoints for doripenem were not available when the study was initiated. Breakpoints for Enterobacteriaceae were subsequently released in June 2010 [8]. CLSI breakpoints were used for imipenem and meropenem [9] since European Committee on Antimicrobial Susceptibility Testing (EUCAST) breakpoints were not available when the study was initiated. However, since EUCAST breakpoints for doripenem, imipenem, and meropenem are now available, they are also used for this data analysis [10].

\section{Results}

Ten centres in Turkey provided 596 eligible isolates. Patient demographics are shown in Table 2. By pathogen group, 297 (49.8\%) were Pseudomonas spp., of which 98.7\% were $P$. aeruginosa (49.2\% of total); 240 (40.3\%) isolates were Enterobacteriaceae, of which $47.9 \%$ were $E$. coli and $35.0 \%$ Klebsiella pneumoniae (19.3\% and $14.1 \%$ of total, respectively); 59 (9.9\%) were other Gram-negative bacteria, of which $89.8 \%$ were $A$. baumannii ( $8.9 \%$ of total) (Table 3).

By type of infection, $42.4 \%$ of isolates were NP; $40.4 \%$, BSI; and $17.1 \%$, cIAI. Slightly more than half of the isolates came from patients in the ICU $(51.8 \%)$, whilst 48.2\% came from non-ICU patients (Table 2).

Of the 596 isolates, 187 (31.4\%) were resistant to at least one carbapenem based on the E-test results reported by the collecting centre. Two hundred fifty-two isolates underwent reference laboratory confirmation of the centres' E-test results. Of the 91 determined by the centres to be susceptible to doripenem using current FDA breakpoints, $73(80.2 \%)$ were confirmed as susceptible by the reference laboratory. Of the 161 determined to be nonsusceptible to doripenem by the centres, 153 (95.0\%) were confirmed as non-susceptible by the reference laboratory. For imipenem, of the 73 determined by the centres to be susceptible using CLSI breakpoints, 68 (93.2\%) were confirmed as susceptible. Of the 179 determined by the

Table 1 Breakpoints

\begin{tabular}{|c|c|c|c|c|c|c|c|c|c|c|c|c|c|c|c|c|c|c|}
\hline \multirow[t]{3}{*}{ Family/Genus (species) } & \multirow{2}{*}{\multicolumn{3}{|c|}{$\frac{\text { FDA }}{\text { Doripenem }}$}} & \multicolumn{6}{|c|}{ CLSI } & \multicolumn{9}{|c|}{ EUCAST } \\
\hline & & & & \multicolumn{3}{|c|}{$\begin{array}{l}\text { Imipenem/ } \\
\text { Meropenem }\end{array}$} & \multicolumn{3}{|c|}{$\begin{array}{c}\text { Doripenem/Imipenem/ } \\
\text { Meropenem* }\end{array}$} & \multicolumn{3}{|c|}{ Doripenem } & \multicolumn{3}{|c|}{ Imipenem } & \multicolumn{3}{|c|}{ Meropenem } \\
\hline & $S$ & $\mathrm{I}$ & $\mathbf{R}$ & $\mathrm{S}$ & 1 & $\mathbf{R}$ & $S$ & 1 & $\mathbf{R}$ & $\mathrm{S}$ & $\mathrm{I}$ & $\mathbf{R}$ & $\mathrm{S}$ & 1 & $\mathbf{R}$ & $S$ & $\mathrm{I}$ & $\mathbf{R}$ \\
\hline Pseudomonas aeruginosa & $\leq 2$ & - & - & $\leq 4$ & 8 & $\geq 16$ & $\leq 4$ & 8 & $\geq 16$ & $\leq 1$ & $2-4$ & $\geq 8$ & $\leq 4$ & 8 & $\geq 16$ & $\leq 2$ & $4-8$ & $\geq 16$ \\
\hline Enterobacteriaceae & $\leq 0.5$ & - & - & $\leq 4$ & 8 & $\geq 16$ & $\leq 1$ & 2 & $\geq 4$ & $\leq 1$ & $2-4$ & $\geq 8$ & $\leq 2$ & $4-8$ & $\geq 16$ & $\leq 2$ & $4-8$ & $\geq 16$ \\
\hline Acinetobacter spp. & $\leq 1$ & - & - & $\leq 4$ & 8 & $\geq 16$ & $\leq 4$ & 8 & $\geq 16$ & $\leq 1$ & $2-4$ & $\geq 8$ & $\leq 2$ & $4-8$ & $\geq 16$ & $\leq 2$ & $4-8$ & $\geq 16$ \\
\hline
\end{tabular}

FDA US Food and Drug Administration; CLSI Clinical and Laboratory Standards Institute; EUCAST European Committee on Antimicrobial Susceptibility Testing; $S$ susceptible; I intermediate; $R$ resistant

*As of June 2010 
Table 2 Isolates from Turkey by patient gender, age, location, and infection type

\begin{tabular}{|c|c|c|c|c|c|c|c|c|c|c|c|c|}
\hline \multirow{2}{*}{$\frac{\text { Number of Isolates }}{\text { Species }}$} & \multicolumn{2}{|c|}{ Gender } & \multicolumn{5}{|c|}{ Age Group } & \multicolumn{2}{|c|}{ Location } & \multicolumn{3}{|c|}{ Infection Type } \\
\hline & $F$ & $M$ & 0 to $2 \mathrm{yrs}$ & 3 to $5 \mathrm{yrs}$ & 6 to $17 \mathrm{yrs}$ & 18 to 64 yrs & $>64$ yrs & ICU & Non-ICU & BSI & clAI & NP \\
\hline Acinetobacter baumannii & 21 & 32 & & & 2 & 36 & 15 & 41 & 12 & 21 & 6 & 26 \\
\hline Acinetobacter haemolyticus & 1 & & & & 1 & & & & 1 & & 1 & \\
\hline Acinetobacter junii/johnsonii & 1 & 2 & & & & 3 & & 3 & & 2 & & 1 \\
\hline Acinetobacter Iwoffii & & 1 & & & & 1 & & & 1 & 1 & & \\
\hline Citrobacter freundii & 1 & & & & & 1 & & & 1 & & 1 & \\
\hline Enterobacter aerogenes & 3 & 6 & & & & 7 & 2 & 6 & 3 & 1 & 2 & 6 \\
\hline Enterobacter cloacae & 2 & 7 & 1 & & & 7 & 1 & 2 & 7 & 3 & 5 & 1 \\
\hline Escherichia coli & 52 & 63 & 3 & 1 & 5 & 54 & 52 & 39 & 76 & 70 & 32 & 13 \\
\hline Klebsiella oxytoca & 4 & 1 & 1 & & 1 & 1 & 2 & 2 & 3 & 3 & & 2 \\
\hline Klebsiella pneumoniae & 33 & 51 & 5 & & 1 & 42 & 36 & 47 & 37 & 38 & 9 & 37 \\
\hline Kluyvera sp. & 1 & 1 & & & & 1 & 1 & 2 & & 1 & 1 & \\
\hline Morganella morganii & 1 & 2 & 1 & & & 1 & 1 & & 3 & 2 & 1 & \\
\hline Pantoea sp. & & 1 & & & & 1 & & & 1 & & 1 & \\
\hline Proteus mirabilis & & 2 & & & & 1 & 1 & 1 & 1 & & 1 & 1 \\
\hline Pseudomonas aeruginosa & 113 & 180 & 18 & 3 & 7 & 168 & 97 & 160 & 133 & 90 & 40 & 163 \\
\hline Pseudomonas putida & 2 & 1 & & & & 1 & 2 & & 3 & 1 & 2 & \\
\hline Pseudomonas stutzeri & & 1 & & & & 1 & & & 1 & & & 1 \\
\hline Raoultella terrigena & 1 & & & & & & 1 & & 1 & 1 & & \\
\hline Serratia marcescens & 2 & 6 & & & 1 & 3 & 4 & 6 & 2 & 7 & & 1 \\
\hline Stenotrophomonas maltophilia & & 1 & & & & 1 & & & 1 & & & 1 \\
\hline Total & 238 & 358 & 29 & 4 & 18 & 330 & 215 & 309 & 287 & 241 & 102 & 253 \\
\hline
\end{tabular}

$B S I$ bloodstream infection; $C I A I$ complicated intra-abdominal infection; $F$ female; ICU intensive care unit; $M$ male; NP nosocomial pneumonia

Table 3 Isolates from Turkey by specimen source

\begin{tabular}{|c|c|c|c|c|c|}
\hline \multirow{2}{*}{$\begin{array}{c}\text { Number of Isolates } \\
\text { Species }\end{array}$} & \multicolumn{5}{|c|}{ Source of Isolation } \\
\hline & Pulmonary Samples & Peritoneal Fluid & Blood & Others & Grand Total \\
\hline Acinetobacter baumannii & 26 & & 21 & 6 & 53 \\
\hline Acinetobacter haemolyticus & & & & 1 & 1 \\
\hline Acinetobacter junii/johnsonii & 1 & & 2 & & 3 \\
\hline Acinetobacter Iwoffii & & & 1 & & 1 \\
\hline Citrobacter freundii & & & & 1 & 1 \\
\hline Enterobacter aerogenes & 6 & & 1 & 2 & 9 \\
\hline Enterobacter cloacae & 1 & 1 & 3 & 4 & 9 \\
\hline Escherichia coli & 13 & 1 & 72 & 29 & 115 \\
\hline Klebsiella oxytoca & 2 & & 3 & & 5 \\
\hline Klebsiella pneumoniae & 37 & & 39 & 8 & 84 \\
\hline Kluyvera sp. & & & 1 & 1 & 2 \\
\hline Morganella morganii & & & 2 & 1 & 3 \\
\hline Pantoea sp. & & & & 1 & 1 \\
\hline Proteus mirabilis & 1 & & & 1 & 2 \\
\hline Pseudomonas aeruginosa & 161 & 1 & 91 & 40 & 293 \\
\hline Pseudomonas putida & & & 1 & 2 & 3 \\
\hline Pseudomonas stutzeri & 1 & & & & 1 \\
\hline Raoultella terrigena & & & 1 & & 1 \\
\hline Serratia marcescens & 1 & & 7 & & 8 \\
\hline Stenotrophomonas maltophilia & 1 & & & & 1 \\
\hline Total & 251 & 3 & 245 & 97 & 596 \\
\hline
\end{tabular}


centres to be non-susceptible (ie, intermediate or resistant) to imipenem, 173 (96.6\%) were confirmed as non-susceptible by the reference laboratory. For meropenem, of the 98 determined by the centres to be susceptible using CLSI breakpoints, 80 (81.6\%) were confirmed as susceptible. Of the 154 determined to be non-susceptible (ie, intermediate or resistant) to meropenem by the centres, 143 (92.9\%) were confirmed as non-susceptible by the reference laboratory.

For $P$. aeruginosa, the $\mathrm{MIC}_{90}$ was lowest for doripenem $(32 \mathrm{mg} / \mathrm{L})$ compared with $\geq 64 \mathrm{mg} / \mathrm{L}$ for both imipenem and meropenem (Table 4). Only $19.5 \%$ of $P$. aeruginosa isolates had a doripenem $\mathrm{MIC}>4 \mathrm{mg} / \mathrm{L}$ compared with $25.8 \%$ and $30.9 \%$ for meropenem and imipenem, respectively (Figure 1, Tables 5, 6, 7). At MIC $2 \mathrm{mg} / \mathrm{L}$ (the FDA breakpoint for doripenem), 64.0\% of Pseudomonas spp. were susceptible to doripenem, $48.2 \%$ to imipenem, and $56.2 \%$ to meropenem. At $\mathrm{MIC} \leq 4 \mathrm{mg} / \mathrm{L}$ (the CLSI breakpoint for imipenem and meropenem), $74.1 \%$ were susceptible to doripenem, $53.9 \%$ to imipenem, and $63.0 \%$ to meropenem.

For Enterobacteriaceae, doripenem and meropenem were equally active $\left(\mathrm{MIC}_{90} 0.12 \mathrm{mg} / \mathrm{L}\right)$ and at least fourfold more active than imipenem $\left(\mathrm{MIC}_{90} 0.5 \mathrm{mg} / \mathrm{L}\right.$; Figure 2). At MIC $0.5 \mathrm{mg} / \mathrm{L}$ (the FDA breakpoint for doripenem against Enterobacteriaceae), 97.5\% were susceptible to doripenem, $93.75 \%$ to imipenem, and $97.5 \%$ to meropenem. At MIC $\leq 4 \mathrm{mg} / \mathrm{L}$ (the 2009 CLSI breakpoint for imipenem and meropenem against Enterobacteriaceae), $98.75 \%$ were susceptible to doripenem, $98.33 \%$ to imipenem, and $98.75 \%$ to meropenem. At

Table 4 Minimum inhibitory concentration (MIC) of all pathogens combined and pathogen groups from Turkey for doripenem, imipenem and meropenem

\begin{tabular}{|c|c|c|c|c|c|}
\hline \multirow[t]{2}{*}{ Turkey } & \multirow[t]{2}{*}{$\mathrm{N}$} & \multicolumn{4}{|c|}{$\mathrm{MIC}(\mathrm{mg} / \mathrm{L})$} \\
\hline & & Minimum & $50 \%$ & $90 \%$ & Maximum \\
\hline All pathogens & 596 & & & & \\
\hline Doripenem & & 0.008 & 0.12 & 32 & $\geq 64$ \\
\hline Imipenem & & 0.06 & 1 & $\geq 64$ & $\geq 64$ \\
\hline Meropenem & & 0.008 & 0.25 & $\geq 64$ & $\geq 64$ \\
\hline Pseudomonas spp. & 297 & & & & \\
\hline Doripenem & & 0.03 & 1 & 32 & $\geq 64$ \\
\hline Imipenem & & 0.12 & 4 & $\geq 64$ & $\geq 64$ \\
\hline Meropenem & & 0.03 & 1 & $\geq 64$ & $\geq 64$ \\
\hline Enterobacteriaceae & 240 & & & & \\
\hline Doripenem & & 0.008 & 0.03 & 0.12 & 32 \\
\hline Imipenem & & 0.12 & 0.25 & 0.5 & $\geq 64$ \\
\hline Meropenem & & 0.008 & 0.03 & 0.12 & $\geq 64$ \\
\hline Other Gram negatives & 59 & & & & \\
\hline Doripenem & & 0.03 & 8 & $\geq 64$ & $\geq 64$ \\
\hline Imipenem & & 0.06 & 32 & $\geq 64$ & $\geq 64$ \\
\hline Meropenem & & 0.06 & 32 & $\geq 64$ & $\geq 64$ \\
\hline
\end{tabular}

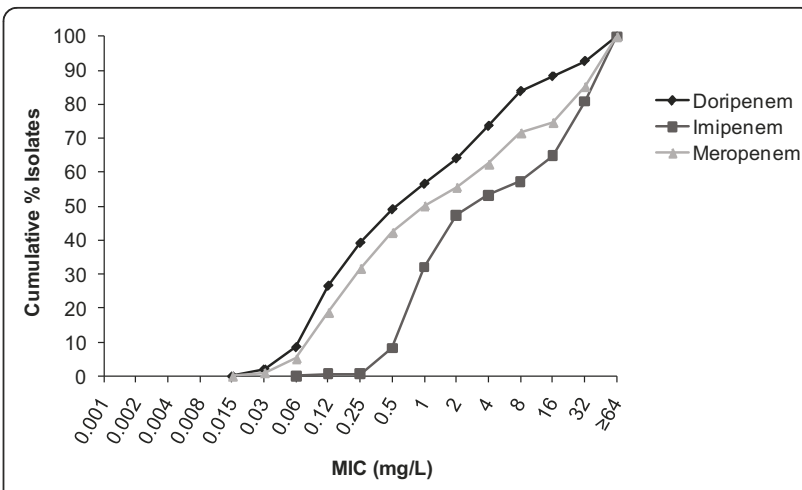

Figure 1 Cumulative\% minimum inhibitory concentration (MIC) distributions against Pseudomonas aeruginosa $(\mathrm{N}=293)$

$\mathrm{MIC} \leq 1 \mathrm{mg} / \mathrm{L}$ (the new breakpoint for imipenem and meropenem, as well as doripenem, against Enterobacteriaceae established by CLSI in June 2010), 97.92\% were susceptible to doripenem, $96.67 \%$ to imipenem and $97.92 \%$ to meropenem. Also at MIC $\leq 1 \mathrm{mg} / \mathrm{L}, 100 \%$ of E. coli and $94.1 \%$ of $K$. pneumoniae were susceptible to each of the three carbapenems. The $\mathrm{MIC}_{90}$ for all three carbapenems against other Gram-negative isolates, including A. baumannii (Figure 3), was $\geq 64 \mathrm{mg} / \mathrm{L}$.

Based on the newly established EUCAST breakpoints for carbapenems (Table 1), 43.4\% of Pseudomonas spp. and $2.1 \%$ of Enterobacteriaceae isolates were deemed doripenem non-susceptible according to the E-test MIC results from the collecting centres (Table 8). Regarding Pseudomonas spp., $46.1 \%$ were non-susceptible to imipenem and $43.8 \%$ to meropenem, whilst $2.1 \%$ and $1.7 \%$ of Enterobacteriaceae were non-susceptible to imipenem and meropenem, respectively.

\section{Discussion}

The COMPACT surveillance study involving 10 centres in Turkey demonstrated that doripenem has similar or slightly better activity than imipenem and meropenem against Pseudomonas spp., Enterobacteriaceae, and other Gram-negative pathogens.

Compared with the other European, Middle Eastern, and African countries that participated in COMPACT, Turkey had a high rate $(31.4 \%)$ of combined imipenem resistance, meropenem resistance, or doripenem non-susceptibility [11]. This rate in Turkey was second only to Russia (33.6\%), and followed by Egypt (28.3\%), Spain (23.6\%), Italy (23.2\%) and the remainder of the 16 countries involved.

In Turkey, doripenem was the most active of the 3 carbapenems against Pseudomonas spp. The non-susceptible (intermediate and resistant) rate of $46.1 \%$ for Pseudomonas spp. (98.7\% P. aeruginosa) to imipenem observed in this study is higher than the $32 \%$ observed 
Table 5 Isolates from Turkey: Doripenem MIC distribution

\begin{tabular}{|c|c|c|c|c|c|c|c|c|c|c|c|c|c|c|c|}
\hline Number of isolates & & & & & Doripe & em E- & est $N$ & $C(\mathrm{~m}$ & & & & & & & \\
\hline Species & 0.008 & 0.015 & 0.03 & 0.06 & 0.12 & 0.25 & 0.5 & 1 & 2 & 4 & 8 & 16 & 32 & $>32$ & Grand Tota \\
\hline Acinetobacter baumannii & & & & & 1 & 1 & 1 & 5 & 3 & 7 & 7 & 3 & 7 & 18 & 53 \\
\hline Acinetobacter haemolyticus & & & & & & 1 & & & & & & & & & 1 \\
\hline Acinetobacter junii/johnsonii & & & 1 & & & & & 1 & 1 & & & & & & 3 \\
\hline Acinetobacter Iwoffii & & & & & & & & & & & & & & 1 & 1 \\
\hline Citrobacter freundii & & 1 & & & & & & & & & & & & & 1 \\
\hline Enterobacter aerogenes & & 3 & 4 & & 2 & & & & & & & & & & 9 \\
\hline Enterobacter cloacae & 1 & 3 & 4 & & 1 & & & & & & & & & & 9 \\
\hline Escherichia coli & 9 & 59 & 37 & 4 & 1 & 3 & 2 & & & & & & & & 115 \\
\hline Klebsiella oxytoca & & & 3 & 2 & & & & & & & & & & & 5 \\
\hline Klebsiella pneumoniae & 2 & 27 & 35 & 10 & 1 & 3 & 1 & & 2 & & 1 & 1 & 1 & & 84 \\
\hline Kluyvera sp. & & 1 & & & & & & 1 & & & & & & & 2 \\
\hline Morganella morganii & & & & 1 & 1 & 1 & & & & & & & & & 3 \\
\hline Pantoea sp. & & & 1 & & & & & & & & & & & & 1 \\
\hline Proteus mirabilis & & 1 & & 1 & & & & & & & & & & & 2 \\
\hline Pseudomonas aeruginosa & & & 6 & 19 & 53 & 37 & 29 & 22 & 22 & 28 & 30 & 13 & 13 & 21 & 293 \\
\hline Pseudomonas putida & & & & & & 1 & & & & 2 & & & & & 3 \\
\hline Pseudomonas stutzeri & & & & & 1 & & & & & & & & & & 1 \\
\hline Raoultella terrigena & & & 1 & & & & & & & & & & & & 1 \\
\hline Serratia marcescens & & & 4 & 1 & 3 & & & & & & & & & & 8 \\
\hline Stenotrophomonas maltophilia & & & & & & & & & & 1 & & & & & 1 \\
\hline Grand Total & 12 & 95 & 96 & 38 & 64 & 47 & 33 & 29 & 28 & 38 & 38 & 17 & 21 & 40 & 596 \\
\hline
\end{tabular}

Table 6 Isolates from Turkey: Imipenem MIC distribution

\begin{tabular}{|c|c|c|c|c|c|c|c|c|c|c|c|c|}
\hline \multirow{2}{*}{$\begin{array}{l}\text { Number of isolates } \\
\text { Species }\end{array}$} & \multicolumn{11}{|c|}{ Imipenem E-test MIC (mg/L) } & \multirow[b]{2}{*}{ Grand Tota } \\
\hline & 0.06 & 0.12 & 0.25 & 0.5 & 1 & 2 & 4 & 8 & 16 & 32 & $>32$ & \\
\hline Acinetobacter baumannii & & & 2 & & 3 & 4 & 3 & 1 & 1 & 15 & 24 & 53 \\
\hline Acinetobacter haemolyticus & & & 1 & & & & & & & & & 1 \\
\hline Acinetobacter junii/johnsonii & 1 & & & & & & 2 & & & & & 3 \\
\hline Acinetobacter Iwoffii & & & & & & & & & & & 1 & 1 \\
\hline Citrobacter freundii & & & & & 1 & & & & & & & 1 \\
\hline Enterobacter aerogenes & & 1 & 5 & 3 & & & & & & & & 9 \\
\hline Enterobacter cloacae & & 1 & 4 & 3 & 1 & & & & & & & 9 \\
\hline Escherichia coli & & 36 & 69 & 9 & 1 & & & & & & & 115 \\
\hline Klebsiella oxytoca & & 1 & 3 & 1 & & & & & & & & 5 \\
\hline Klebsiella pneumoniae & & 26 & 44 & 7 & 2 & & 1 & 1 & & 2 & 1 & 84 \\
\hline Kluyvera sp. & & & 1 & & & 1 & & & & & & 2 \\
\hline Morganella morganii & & & & & 1 & 2 & & & & & & 3 \\
\hline Pantoea sp. & & & & 1 & & & & & & & & 1 \\
\hline Proteus mirabilis & & 1 & & & 1 & & & & & & & 2 \\
\hline Pseudomonas aeruginosa & & 2 & & 22 & 70 & 45 & 17 & 12 & 22 & 47 & 56 & 293 \\
\hline Pseudomonas putida & & & 1 & & 2 & & & & & & & 3 \\
\hline Pseudomonas stutzeri & & & & 1 & & & & & & & & 1 \\
\hline Raoultella terrigena & & & 1 & & & & & & & & & 1 \\
\hline Serratia marcescens & & & 4 & 4 & & & & & & & & 8 \\
\hline Stenotrophomonas maltophilia & & & & & & & & & & 1 & & 1 \\
\hline Grand Total & 1 & 68 & 135 & 51 & 82 & 52 & 23 & 14 & 23 & 65 & 82 & 596 \\
\hline
\end{tabular}


Table 7 Isolates from Turkey: Meropenem MIC distribution

\begin{tabular}{|c|c|c|c|c|c|c|c|c|c|c|c|c|c|c|c|}
\hline Number of isolates & & & & & Merope & nem $E$ - & est $n$ & $C(\mathrm{~m}$ & & & & & & & \\
\hline Species & 0.008 & 0.015 & 0.03 & 0.06 & 0.12 & 0.25 & 0.5 & 1 & 2 & 4 & 8 & 16 & 32 & $>32$ & Grand Tota \\
\hline Acinetobacter baumannii & & & & & & 1 & 1 & 4 & 3 & 5 & 5 & 5 & 8 & 21 & 53 \\
\hline Acinetobacter haemolyticus & & & & & 1 & & & & & & & & & & 1 \\
\hline Acinetobacter junii/johnsonii & & & & 1 & & & & & 1 & & & 1 & & & 3 \\
\hline Acinetobacter Iwoffii & & & & & & & & & & & & & & 1 & 1 \\
\hline Citrobacter freundii & & 1 & & & & & & & & & & & & & 1 \\
\hline Enterobacter aerogenes & & 3 & 4 & 1 & 1 & & & & & & & & & & 9 \\
\hline Enterobacter cloacae & 1 & 1 & 6 & & 1 & & & & & & & & & & 9 \\
\hline Escherichia coli & 3 & 50 & 39 & 16 & 3 & 2 & 2 & & & & & & & & 115 \\
\hline Klebsiella oxytoca & & 1 & 2 & 2 & & & & & & & & & & & 5 \\
\hline Klebsiella pneumoniae & 1 & 20 & 32 & 18 & 4 & 2 & 2 & & 1 & 1 & & & 2 & 1 & 84 \\
\hline Kluyvera sp. & & 1 & & & & & & 1 & & & & & & & 2 \\
\hline Morganella morganii & & & 1 & 1 & 1 & & & & & & & & & & 3 \\
\hline Pantoea sp. & & 1 & & & & & & & & & & & & & 1 \\
\hline Proteus mirabilis & & & 1 & & 1 & & & & & & & & & & 2 \\
\hline Pseudomonas aeruginosa & & & 3 & 12 & 40 & 38 & 31 & 23 & 16 & 20 & 27 & 9 & 31 & 43 & 293 \\
\hline Pseudomonas putida & & & & & & & & 2 & 1 & & & & & & 3 \\
\hline Pseudomonas stutzeri & & & & & & 1 & & & & & & & & & 1 \\
\hline Raoultella terrigena & & & 1 & & & & & & & & & & & & 1 \\
\hline Serratia marcescens & & 1 & 2 & 3 & 1 & 1 & & & & & & & & & 8 \\
\hline Stenotrophomonas maltophilia & & & & & & & & & 1 & & & & & & 1 \\
\hline Grand Total & 5 & 79 & 91 & 54 & 53 & 45 & 36 & 30 & 23 & 26 & 32 & 15 & 41 & 66 & 596 \\
\hline
\end{tabular}

from 2004 to 2006 with VAP isolates [12]. The non-susceptible rate for imipenem in this study also is higher than the $16.1 \%$ observed from 2000 to 2002 for P. aeruginosa in both coronary and surgical ICU patients [13].

Against Enterobacteriaceae, doripenem and meropenem were equally active and at least four-fold more active than imipenem. These results are consistent with susceptibility data from the United Kingdom and Ireland for 2001 to 2006 [14]. In addition, the activity of imipenem and meropenem observed in Turkey in COMPACT

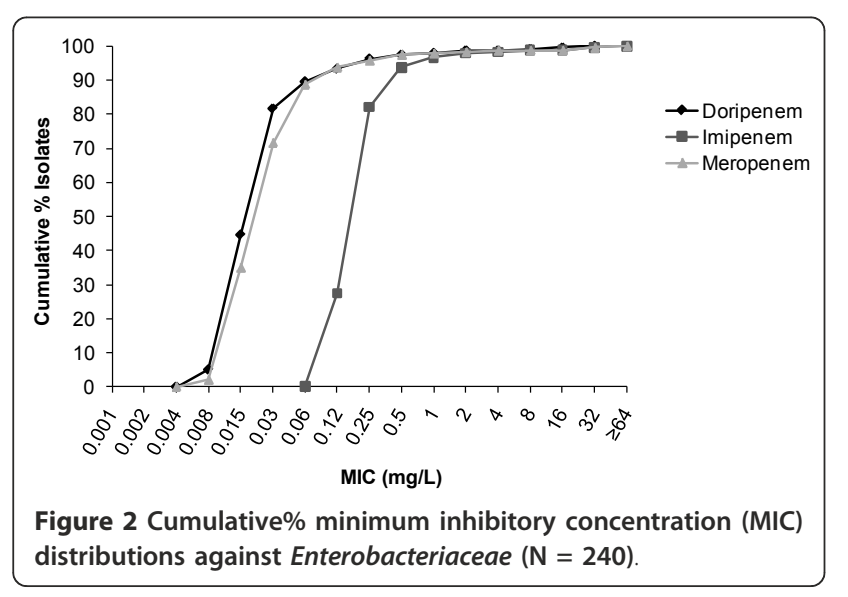

was very similar to the susceptibility rate of $97.6 \%$ observed by Korten et al. for imipenem against all Enterobacteriaceae isolates from Turkey between 2000 and 2003 [15].

None of the 3 carbapenems showed good activity against A. baumannii. This is not surprising given the high rates of resistance observed over the past decade in Turkey $[12,13]$. The $\mathrm{MIC}_{90}$ for $A$. baumannii was several-fold higher in this study than the $\mathrm{MIC}_{90}>8$ and $>16 \mathrm{mg} / \mathrm{L}$ observed for imipenem and meropenem, respectively, in

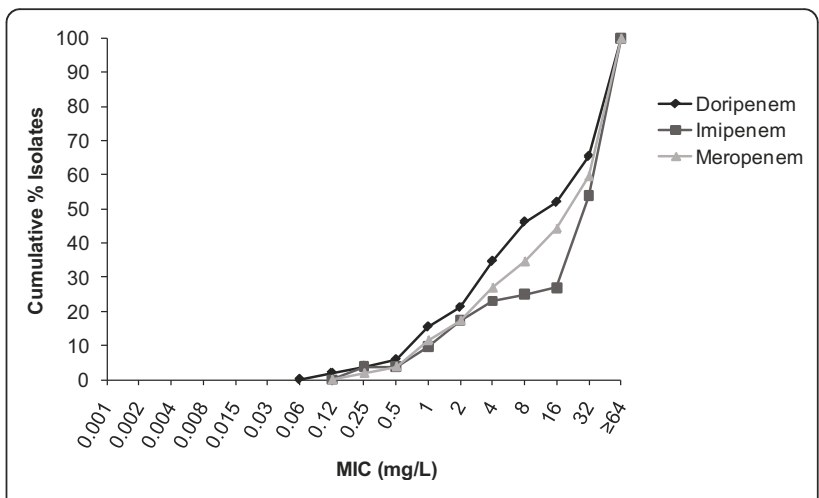

Figure 3 Cumulative\% minimum inhibitory concentration (MIC) distributions against Acinetobacter baumannii $(\mathrm{N}=53)$. 
Table 8 Susceptibility* of all pathogens from Turkey: Combined pathogens and pathogen groups for doripenem, imipenem, and meropenem

\begin{tabular}{lcccc}
\hline \multirow{2}{*}{ Turkey } & $\mathbf{N}$ & \multicolumn{3}{c}{ Percentage of isolates } \\
\cline { 2 - 5 } & & Susceptible & Intermediate & Resistant \\
\hline All pathogens & 596 & & 11.1 & 19.5 \\
Doripenem & & 69.5 & 3.4 & 28.5 \\
Imipenem & & 68.1 & 9.7 & 20.5 \\
Meropenem & & 69.8 & & \\
\hline Pseudomonas spp. & 297 & & 17.5 & 25.9 \\
Doripenem & & 56.6 & 4.0 & 42.1 \\
Imipenem & & 53.9 & 15.8 & 28.0 \\
Meropenem & 56.2 & 0.8 & 1.3 \\
\hline Enterobacteriaceae & 240 & & 0.8 & 1.3 \\
Doripenem & & 97.9 & 0.4 & 1.3 \\
Imipenem & & 97.9 & & \\
Meropenem & 98.3 & 20.3 & 61.0 \\
\hline Other Gram negatives & 59 & & 10.2 & 71.2 \\
Doripenem & & 18.6 & 17.0 & 61.0 \\
Imipenem & & 18.6 & \\
Meropenem & & 22.0 &
\end{tabular}

*Based on current European Committee on Antimicrobial Susceptibility Testing breakpoints

the SENTRY Antimicrobial Surveillance Program from 2000 to 2006 in Ankara and Istanbul, Turkey [3].

The COMPACT surveillance study also was carried out in 6 Asia-Pacific countries. As in the 10 centres in Turkey and the 80 centres throughout Europe, the Middle East, and Africa, doripenem was the most active of the carbapenems tested against Asia-Pacific isolates [16]. The $\mathrm{MIC}_{90}$ against all Asia-Pacific isolates was $8 \mathrm{mg} / \mathrm{L}$ for doripenem compared with $32 \mathrm{mg} / \mathrm{L}$ for Turkey. The mean $\mathrm{MIC}_{90}$ against all isolates for imipenem and meropenem (both $\geq 64 \mathrm{mg} / \mathrm{L}$ ) was the same for Turkey and the Asian-Pacific countries.

\section{Conclusions}

In conclusion, the carbapenems possess good activity against the Gram-negative isolates included in this study, including Pseudomonas spp. and Enterobacteriaceae, among the 10 collecting centres in Turkey. Although the rate of combined imipenem resistance, meropenem resistance, or doripenem non-susceptibility was high in Turkey and second only to Russia, doripenem was the most active carbapenem against $P$. aeruginosa, was equally active to meropenem, and was more active than imipenem against Enterobacteriaceae.

\section{Author details}

'Ondokuzmayis University, Kurupelit Kampüsü, PK 55139, Kurupelit, Samsun, Turkey. ${ }^{2}$ Dokuz Eylül University Infectious Disease Clinic, 35340, İnciralti İZMIR, Izmir, Turkey. ${ }^{3}$ Dicle University Infectious Disease Clinic, 21280, Diyarbakir, Turkey. ${ }^{4}$ Ankara University Infectious Disease Clinic, PK 06230,
Altindağ, Gündoğdu Ankara, Turkey. ${ }^{5}$ Janssen-Turkey, Ertürk Sok, Keçeli Plaza No: 13 34810, Kavacik, Beykoz, Istanbul, Turkey. ' Janssen-EMEA,

Sihlbruggstrasse 111, 6340, Baar, Switzerland.

\section{Authors' contributions}

HL was involved in the trial design as well as data collection and analysis He also participated in drafting the manuscript, and reviewed and approved the final draft. NC was involved in the data collection and analysis. He also participated in drafting the manuscript, and reviewed and approved the final draft. MC was involved in the data collection and analysis. He also participated in drafting the manuscript, and reviewed and approved the final draft. HK was involved in the data collection and analysis. He also participated in drafting the manuscript and reviewed the final draft. HB was involved in the trial design and data analysis. He also participated in drafting the manuscript, and reviewed and approved the final draft. $J$ was involved in the trial design and data analysis. He also participated in drafting the manuscript, and reviewed and approved the final draft. All authors read and approved the final manuscript.

COMPACT Turkey study investigators

F. Akata and Z. Yuluğkural (Trakya University, Edirne, Turkey); C. Ayaz (Dicle University, Diyarbakir, Turkey); G. Çelebri and F. Cömert (Karaelmas University, Zonguldak, Turkey); D. Gerçeker (Ankara University, Ankara, Turkey); Z. Gülay (Dokuz Eylül University, Izmir, Turkey); M. Günaydin (Ondokuzmayis University, Samsun, Turkey); A. Kaya and G. Ersöz (Mersin University, Mersin, Turkey); S. Özer and N. Benzononana (Dr. Lütfü Kardar Hospital, Istanbul, Turkey); and Y. Taşova and A. Yaman (Çukorova University, Adana, Turkey).

\section{Competing interests}

This work was supported by Janssen EMEA. The decision to submit this article for publication was made by Janssen and the authors. No financial support or honorarium was given to the non- Janssen authors for the development of this manuscript. H. Baris and J. Laeuffer are employees of Janssen EMEA. These authors were not awarded any additional support outside of their salaries for their participation in this study. Editorial assistance was provided by Phase Five Communications Inc., which was funded by Janssen EMEA.

Received: 16 June 2011 Accepted: 16 February 2012

Published: 16 February 2012

\section{References}

1. Health Protection Agency: Surveillance of healthcare associated infections report. [http://www.hpa.org.uk/web/HPAwebFile/HPAweb_C/ 1216193833496]

2. Turner PJ: MYSTIC Europe 2007: activity of meropenem and other broadspectrum agents against nosocomial isolates. Diagn Microbiol Infect Dis 2009, 63:217-222.

3. Gur D, Korten V, Unal S, Deshpande LM, Castanheira M: Increasing carbapenem resistance due to the clonal dissemination of oxacillinase (OXA-23 and OXA-58)-producing Acinetobacter baumannii: report from the Turkish SENTRY Program sites. J Med Microbiol 2008, 57:1529-1532.

4. Jones RN, Huynh HK, Biedenbach DJ, Fritsche TR, Sader HS: Doripenem (S4661), a novel carbapenem: comparative activity against contemporary pathogens including bactericidal action and preliminary in vitro methods evaluations. J Antimicrob Chemother 2004, 54:144-154.

5. Doribax: Annex I. Summary of product characteristics. European Medicines Agency [http://www.ema.europa.eu/docs/en_GB/document_library/EPAR__Product_Information/human/000891/WC500037148.pdf].

6. Doribax [package insert]. Ortho-McNeil: Raritan, NJ; 2009.

7. Clinical and Laboratory Standards Institute: Methods for Dilution Antimicrobial Susceptibility Tests for Bacteria That Grow Aerobically. 8 edition. Wayne, PA; 2009.

8. Clinical and Laboratory Standards Institute: Performance Standards for Antimicrobial Susceptibility Testing; Twentieth Informational Supplement (June 2010 Update) Wayne, PA; 2010.

9. Clinical and Laboratory Standards Institute: Performance Standards for Antimicrobial Susceptibility Testing. 19 edition. Wayne, PA; 2009.

10. European Committee on Antimicrobial Susceptibility Testing: Breakpoint tables for interpretation of MICs and zone diameters. Version 1.2.[http:// www.eucast.org/]. 
11. Nordmann P, Picazo JJ, Mutters R, Korten V, Quintana A, Laeuffer JM, Seak JC, Flamm RK, Morrissey I, The COMPACT study group: The Comparative Activity of Carbapenem Testing (COMPACT) study. J Antimicrob Chemother 2011, 66:1070-1078.

12. Erdem I, Ozgultekin A, Inan AS, Dincer E, Turan G, Ceran N, Engin DO, Akcay SS, Akgun N, Goktas P: Incidence, etiology, and antibiotic resistance patterns of Gram-negative microorganisms isolated from patients with ventilator-associated pneumonia in a medical-surgical intensive care unit of a teaching hospital in Istanbul, Turkey (2004-2006). Jpn J Infect Dis 2008, 61:339-342.

13. Kucukates E: Antimicrobial resistance among Gram-negative bacteria isolated from intensive care units in a cardiology institute in Istanbul, Turkey. Jpn J Infect Dis 2005, 58:228-231.

14. Livermore DM, Hope R, Brick G, Lillie M, Reynolds R, The BSAC Working Parties on Resistance Surveillance: Non-susceptibility trends among Enterobacteriaceae from bacteraemias in the UK and Ireland, 2001-06. J Antimicrob Chemother 2008, 62(suppl 2):ii41-ii54.

15. Korten V, Ulusoy S, Zarakolu P, Mete B, Turkish MYSTIC Study Group: Antibiotic resistance surveillance over a 4-year period (2000-2003) in Turkey: results of the MYSTIC Program. Diagn Microbiol Infect Dis 2007, 59:453-457.

16. Christiansen K, Ip M, Ker HB, Mendoza M, Hsu L, Kiratisin P, Chongthaleong A, Redjeki IS, Quintana A, Flamm R, Garcia J, Cassettari M, Cooper D, Okolo P, Morrissey I: In vitro activity of doripenem and other carbapenems against contemporary Gram-negative pathogens isolated from hospitalised patients in the Asia-Pacific region: results of the COMPACT Asia-Pacific Study. Int J Antimicrob Agents 2010, 36:501-506.

\section{Pre-publication history}

The pre-publication history for this paper can be accessed here: http://www.biomedcentral.com/1471-2334/12/42/prepub

doi:10.1186/1471-2334-12-42

Cite this article as: Leblebicioglu et al:: Comparative activity of carbapenem testing (the COMPACT study) in Turkey. BMC Infectious Diseases 2012 12:42.

\section{Submit your next manuscript to BioMed Central and take full advantage of:}

- Convenient online submission

- Thorough peer review

- No space constraints or color figure charges

- Immediate publication on acceptance

- Inclusion in PubMed, CAS, Scopus and Google Scholar

- Research which is freely available for redistribution

Submit your manuscript at www.biomedcentral.com/submit
Biomed Central 